\title{
An Indentation Model for Sandwich Beams with Functional Graded Metallic Foam Core
}

\author{
L. $\mathrm{Mu}$ \\ State Key Laboratory of Mechanical Structure Strength and \\ Vibration, School of Aerospace \\ Xi'an Jiaotong University \\ Xi'an 710049, P.R. China
}

\author{
D.B. Xiao \\ State Key Laboratory of Mechanical Structure Strength and \\ Vibration, School of Aerospace \\ Xi'an Jiaotong University \\ Xi'an 710049, P.R. China
}

\author{
G.P. Zhao* \\ State Key Laboratory of Mechanical Structure Strength and Vibration, School of Aerospace, \\ Xi'an Jiaotong University, \\ Xi'an 710049, P.R. China \\ *Corresponding author
}

\begin{abstract}
Sandwich beams with functional gradient metal foam core are sensitive to local indention because of the low strength of the foam core and the low bending stiffness of the thin face sheets. The local indentation response of the sandwich beams are determined by loading the beams with a flat indenter. The sandwich beam is modelled as an infinite length, isotropic, plastic membrane on a rigid-plastic foundation. By using the principle of virtual velocities, concisely explicit theoretical solutions for the indentation forces and shape functions of deformed zones of the sandwich beams were derived. The analytical results were verified by numerical simulation results. The influence of the gradient foam core on the localized behaviour of the sandwich beam was discussed.
\end{abstract}

Keywords-indentation; sandwich beam; gradient metal foam

\section{INTRODUCTION}

Metallic sandwich structures are widely used in the aviation industry as well as in ship and railway engineering because of their low density, high specific strength, and effective energy absorption. The sandwich structures comprise commonly porous material cores, such as foams, honeycombs, or truss lattices. Natural porous structures are often gradient, meaning that the porosity is not uniform. It may, therefore, be of interest to study such gradient metal foam as well.

Recently, the study of various structures with gradient foam cores has attracted much attention[1]. The use of gradient foam cores in sandwich structures can change structural flexural bearing capacity as required, but the improvement strongly depends on the indentation involved in the deformation. So it is very meaningful to discuss the indentation behaviour of sandwich beams with gradient foam core.

Researchers have studied on the indentation behaviour of sandwich structures with homogeneous core. Some analytical models focused on elastic response of the whole sandwich structures [2,3], while some others studied the sandwich structures which is composed of elastic or plastic face sheets and plastic foam core $[4,5,6]$.

Although many researchers have investigated the indentation behavior of sandwich structures, there is little description of the local indentation of sandwich structures with gradient metallic foam cores. In this study, based on the principle of virtual velocity, the plastic indentation response of sandwich beams with gradient metallic foam core is determined by loading the beams with a flat indenter. The theoretical solutions for the indentation forces and shape functions of deformed zones of the sandwich beams are derived. This model is validated with finite element numerical analysis.

\section{STRUCTURAL MODEL}

Consider sandwich beams with continuous gradient metallic foam core indented by a rigid flat indenters, as represented schematically in fig. 1 . The beam with width $b$ has an infinite length and is composed of two face sheets and a gradient foam core with thicknesses $h$ and $c$, respectively. The flow stress of the face sheet material is $\sigma_{0}$. The foam core has a gradient plateau stress $\sigma_{c}$. The indentation displacement of the indenter is denoted by $\delta$, while deflection profile of the dented zone is represented by $w(x)$. The length of the deformed region is denoted by $2 \xi$. The width of the flat indenter is $2 R$, as marked in fig. 1 .

In the present model, elastic response of the sandwich beam is neglected. The upper face sheet is modelled as an infinite length, ideally plastic thin beam resting on a rigidplastic foundation, which gives a gradient crushing resistance by the graded foam core.

\section{THE MATERIAL PROPERTIES}

In the manuscript, the foam core has a gradient plateau stress $\sigma_{c}$, which can be calculated by 


$$
\sigma_{c}=\frac{\sigma_{c b}-\sigma_{c t}}{C} w(x)+\sigma_{c t}
$$

Where $c$ is the thickness of gradient foam core, $\sigma_{c t}$ and $\sigma_{c b}$ are the constant plateau stress of the gradient foam core on the top and on the bottom, respectively.

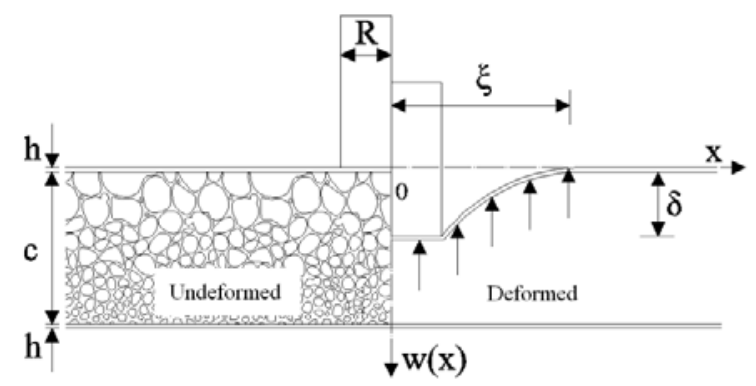

FIGURE 1: SCHEMATIC PROFILES OF UNDEFORMED AND DEFORMED ZONES OF THE SANDWICH BEAMS WITH GRADIENT CORE LOADING WITH A FLAT INDENTER.

\section{LOAD-INDENTATION CHARACTERISTICS}

The principle of virtual velocities is employed to investigate the localized indentation behavior of the sandwich beam. The statement of energy equilibrium requires that the rate of external work equals to the total rate of internal energy dissipation, i.e.,

$$
\dot{W}_{\text {ext }}=\dot{W}_{\text {int }}
$$

The rate of work by external force is

$$
\dot{W}_{e x t}=P \dot{\delta}
$$

Where $P$ is the indentation force and $\dot{\delta}$ is the virtual velocity of the indenter. For the gradient foam core of the sandwich beam $\left(\sigma_{c b} \geq \sigma_{c t}\right)$ indented by a flat indenter, the velocity field of the dented zone and the displacement field of the deformation zone are assumed as [5].

$$
\begin{gathered}
\dot{w}(x, t)= \begin{cases}\dot{\delta}, & 0 \leq|x| \leq R \\
\dot{\delta} \frac{\xi-|x|}{\xi-R}, & R \leq|x| \leq \xi\end{cases} \\
w(x, t)= \begin{cases}\delta, & 0 \leq|x| \leq R \\
\delta\left(\frac{\xi-|x|}{\xi-R}\right)^{2}, & R \leq|x| \leq \xi\end{cases}
\end{gathered}
$$

Where $t$ represents the time.

The strain rate of the upper face sheet is defined according to the theory of moderately large deflection of the beam.

$$
\dot{\varepsilon}_{x}=\frac{d w}{d x} \frac{d \dot{w}}{d x}
$$

For the sandwich beam indented by a flat indenter, the contribution to the rate of internal energy dissipation by membrane force in the dented region can be derived from

$$
\dot{W}_{1}=\int_{A} N_{0} \dot{\varepsilon}_{x} d A=2 \int_{0}^{\xi} N_{0} \dot{\varepsilon}_{x} b d x=\frac{2 \sigma_{0} b h \delta \dot{\delta}}{\xi-R}
$$

Where $N_{0}=\sigma_{0} h$. The contribution to the rate of energy dissipation due to compressive deformation of the foam core can be calculated by

$$
\begin{aligned}
\dot{W}_{2} & =\int_{V} \sigma_{c} d \dot{V} \\
& =2 \dot{\delta} b R\left(\frac{\sigma_{c b}-\sigma_{c t}}{c} \delta+\sigma_{c t}\right)+\dot{\delta} b(\xi-R)\left(\frac{1}{2} \frac{\sigma_{c b}-\sigma_{c t}}{c} \delta+\sigma_{c t}\right)
\end{aligned}
$$

The rate of internal work of the sandwich beam is mainly given by the sum of contribution due to extensional deformation of face sheets and compressive deformation of the foam core. The total rate of internal energy dissipation,

$$
\begin{aligned}
\dot{W}_{\text {int }}= & \dot{W}_{1}+\dot{W}_{2} \\
& \frac{2 \sigma_{0} b h \dot{\delta} \dot{\delta}}{\xi-R}+2 \dot{\delta} b R\left(\frac{\sigma_{c b}-\sigma_{c t}}{c} \delta+\sigma_{c t}\right)+\dot{\delta} b(\xi-R)\left(\frac{1}{2} \frac{\sigma_{c b}-\sigma_{c t}}{c} \delta+\sigma_{c t}\right)
\end{aligned}
$$

Submitting equations (3) and (9) in equation (2), we have

$$
P=\frac{2 \sigma_{0} b h \delta}{\xi-R}+2 b R\left(\frac{\sigma_{c b}-\sigma_{c t}}{c} \delta+\sigma_{c t}\right)+b(\xi-R)\left(\frac{1}{2} \frac{\sigma_{c b}-\sigma_{c t}}{c} \delta+\sigma_{c t}\right)
$$

Based on the principle minimum work, it is required that the half length of deformed region $\xi$ should satisfy the condition of the minimum force for a given indentation, i.e., $\partial P / \partial \xi=0$. This leads to a relationship between $\xi$ and $\delta$ by the following formula.

$$
\xi=R+\left(\frac{4 \sigma_{0} h \delta c}{\left(\sigma_{c b}-\sigma_{c t}\right) \delta+2 \sigma_{c t} c}\right)^{1 / 2}
$$

\section{MODELLING THE INDENTATION BEHAVIOUR OF SANDWICH STRUCTURES}

For validating the gradient sandwich model presented in above section, we modelled the indenter as a rigid body, while the foam core and the face sheets were simulate using 8-node linear brick (C3D8R) element and four-node shell elements(S4R) with reduced integration, respectively. In simulation, the gradient foam core is divided into 10 layers and the thickness of each layer is the same. The lower and edge boundary of the sandwich beams were fully clamped.

The mechanical properties of the face sheet material are following: $E=200 \mathrm{GPa}$,Poisson ratio $v=0.3$ and the engineering stress-strain hardening data are cited from reference [7].

The flow stress and Young's modulus of matrix material of the foam are $\sigma_{0, s}=111.4 \mathrm{MPa}$ and $E_{s}=94.1 \mathrm{GPa}$, respectively. The mechanical properties of the foam core for 
various relative densities are cited from reference[8]. The relative densities of each layer in the core are shown in the table 1 and the numerical results are shown in the fig. 2 .

TABLE 1: THE RELATIVE DENSITY OF LAYERS OF THE GRADIENT FOAM CORE IN NUMERICAL ANALYSIS (10 LAYERS).

\begin{tabular}{|c|c|c|c|c|c|c|c|c|c|c|}
\hline $\begin{array}{c}\text { Nth } \\
\text { layer }\end{array}$ & 1 & 2 & 3 & 4 & 5 & 6 & 7 & 8 & 9 & 10 \\
\hline$\rho_{C}$ & & & & & & & & & & \\
( & 10 & 16 & 21 & 27 & 32 & 37 & 43 & 48 & 54 & 59 \\
& & & & \\
$\mathrm{~kg} / \mathrm{m}^{3}$ & 8 & 2 & 6 & 0 & 4 & 8 & 2 & 6 & 0 & 4 \\
) & & & & & & & & & & \\
\hline
\end{tabular}

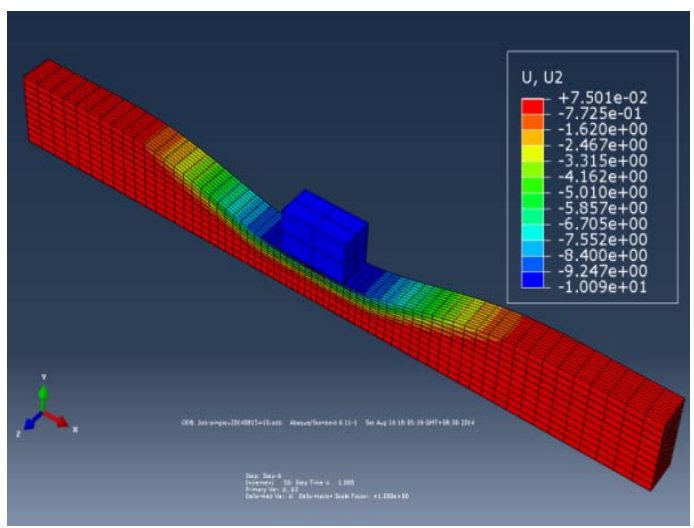

FIGURE 2: DEFORMATION OF THE SANDWICH BEAM WITH 10 LAYERS CORE UNDER A FLAT INDENTER.

\section{RESULTS AND DISCUSSION}

We introduce two dimensionless parameters

$$
\varphi_{c b}=\frac{\sigma_{c b} R}{\sigma_{0} h} \quad \varphi_{c t}=\frac{\sigma_{c t} R}{\sigma_{0} h}
$$

Which are related to the ability of the resistance of the sandwich beam to localized indentation deformation. These dimensionless parameters are usually small due to the low strength of the foam core material. Some other dimensionless parameters are defined as

$$
\begin{gathered}
\bar{x}=\frac{x}{R}, \bar{\xi}=\frac{\xi}{R}, \bar{w}=\frac{w}{R}, \bar{\delta}=\frac{\delta}{R}, \bar{c}=\frac{c}{R}, \\
\bar{p}=\frac{p}{\sigma_{0} b h} \\
\bar{\xi}=1+\left(\frac{4 \bar{\delta} \bar{c}}{\left(\varphi_{c b}-\varphi_{c t}\right) \bar{\delta}+2 \varphi_{c b} \bar{c}}\right)^{1 / 2} \\
\bar{p}=\left(\frac{\left(\varphi_{c b}-\varphi_{c t}\right) \bar{\delta}^{2}+2 \varphi_{c t} \bar{c} \bar{\delta}}{\bar{c}}\right)^{1 / 2}+2\left(\frac{\varphi_{c b}-\varphi_{c t}}{\bar{c}} \bar{\delta}+\varphi_{c t}\right) \\
+\left(\frac{4 \bar{\delta} \bar{c}}{\left(\varphi_{c b}-\varphi_{c t}\right) \bar{\delta}+2 \varphi_{c t} \bar{c}}\right)^{1 / 2}\left(\frac{1}{2} \frac{\varphi_{c b}-\varphi_{c t}}{\bar{c}} \bar{\delta}+\varphi_{c t}\right)
\end{gathered}
$$

The theoretical and simulated results for the case of flat indenter with $\varphi_{c t}=\varphi_{c b}=0.0433$ are shown in the fig.3. The theoretical predictions agree well with the simulated results when the foam core is uniform. The results for the case with $\varphi_{c t}=0.0074, \varphi_{c b}=0.0954$ are also shown in the fig.3. There is a little bit of error, but acceptable, between the theoretical predictions and numerical simulation. The main reason is that the foam core used in numerical simulation is 10 layers with stratified constant-gradient instead of the core with linear gradient.

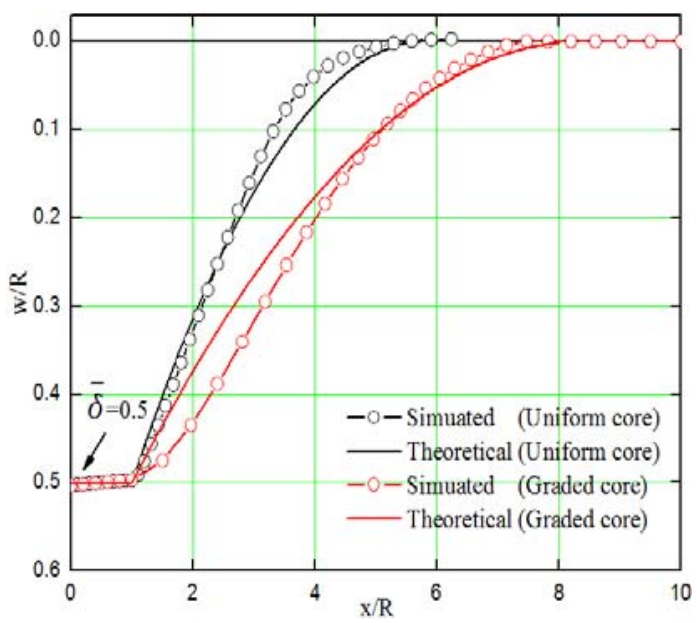

FigURE 3: Distribution OF FACE SHEET DEFORMATION FOR $\bar{\delta}=0.5$.

Fig.4(a) shows that with the increase of the difference of foam densities between the top and the bottom, in which the mass of the foam core is constant, the radius of the deformed region appears increased, in another word, the deformation of the sandwich beams is more obvious. Fig.4(b) shows that with the increase of the difference of foam densities between the top and the bottom, the indenter force is relatively smaller and smaller in initial phase. As the indentation depth and the deformed region expand, the effect of the difference of foam densities between the top and the bottom gradually decrease. Finally, the situation is going into reverse.

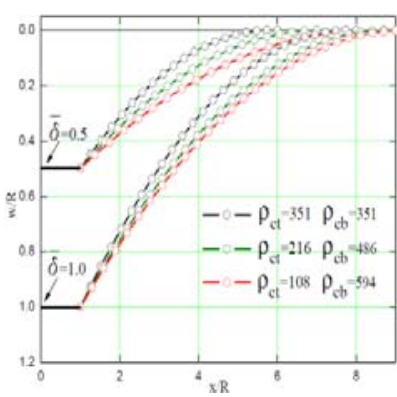

(a)

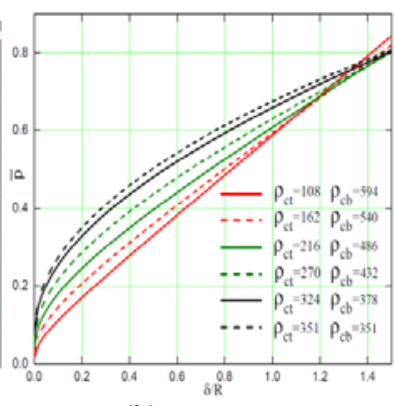

(b)
FIGURE 4: (A) DISTRIBUTION OF DEFORMATION AND (B) LOADINDENTATION CURVES FOR VARIOUS GRADIENT FOAMS

\section{CONCLUSIONS}

The indentation behaviour of sandwich beams with gradient metal foam core is analyzed using the principle of virtual velocities. In the analytical models, the influence of 
gradient foam core on the local indentation response of the sandwich beams is represented by the two dimensionless characteristic parameters $\varphi_{c t}$ and $\varphi_{c b}$. A 3D finite element model is established using numerical analysis to verify the validity of the analytical solutions. The numerical results are in good agreement with analytical solutions. The influence of the gradient foam core on the load-indentation curves and profiles of the dented zone are also discussed. With the analytic methods being extended, the sandwich structures with more complex gradient foam core will be studied.

\section{ACKNOWLEDGEMENTS}

This work is supported by the National Natural Science Foundation of China (11372237) and the Natural Science Foundation of Shaanxi Province (2011JM1012).

\section{REFERENCES}

[1] Arnaud, P., Yves, C., Laurent, P., Andreas, M., Graded open-cell aluminium foam core sandwich beams. Materials Science and Engineering, 404 , pp. 9-18, 2005.

[2] Du, L., Jiao, G.Q., Indentation study of Z-pin reinforced polymer foam core sandwich structures. Composites: Part A, 40, pp.822-829, 2009.

[3] Turk, M.H., Fatt, M.S.H., Localized damage response of composite sandwich plates. Composites: Part B, 30, pp.157-165, 1999.

[4] Koissin, V., Shipsha, A., Rizov, V., The inelastic quasi-static response of sandwich structures to local loading. Composite Structures, 64, pp. 129-138, 2004.

[5] Xie, Z.Y, Zheng, Z.J., Yu, J.L., Localized indentation of sandwich beam with metallic foam core. Journal of Sandwich Structures and Materials, 14(2), pp.197-210, 2011.

[6] Xie, Z.Y, Zheng, Z.J., Yu, J.L., Localized indentation of sandwich panels with metallic foam core: Analytical models for two types of indenters. Composites: Part B, 44, pp.212-217, 2013.

[7] Santosa, S., Banhart, J., Wierzbicki, T., Experimental and numerical analyses of bending of foam-filled sections. Acta Mechanica, 148, pp. 199-213, 2001.

[8] Gibson, L.J., Ashby, M.F., Cellular solids: structure and properties, Cambridge University Press, 1997. 